\title{
Discussion of Chinese Grammatical Features and Modernization of Chinese Linguistics
}

\author{
Li Ran \\ Shaanxi Polytechnic Institute \\ Xianyang Shaanxi 712000
}

\begin{abstract}
As a witness to the history of the Chinese nation, Chinese has a very rich historical connotation, and thus it is needed to have a better understanding of Chinese grammatical features and corresponding targeted teaching measures in the process of understanding and studying Chinese. Therefore, based on previous studies of Chinese grammatical features and the construction of Chinese language subject, the author summarizes the grammatical forms and characteristics of Chinese and also clarifies the related problems in the modernization of modern Chinese linguistics, such as deficiencies in the cultural inheritance and lack of specific understanding in the integrated Chinese-western linguistics development. The author believes that the cultural and educational goals and methods should be innovated on this basis, so as to highlight the modernization of Chinese language disciplines, enhance the vitality and innovation of the development of Chinese linguistics and promote the continuous development of Chinese language.
\end{abstract}

Keywords-Chinese grammatical feature; modernization of Chinese language teaching; discussion

\section{INTRODUCTION}

Modern Chinese is the most widely used language in the Sino-Tibetan, actually, it is the Mandarin of the Han nationality. Compared with the western language system, Chinese has its own characteristics in the word order, vocabulary, grammar and other aspects. Chinese also has its own characteristics in the grammar structure. At the same time, the language features of Chinese are very distinct because of the large amount vocabulary, the changeable sentence structures, the diversiform usage of function words and different forms of words [1]. Today, with the development of modern culture research and social economy, Chinese linguistics has gained momentum of continuous development as a necessary science to study and understand Chinese. The studies of Chinese language based on grammatical features are not a few and the study of grammar is conducive to making the structure of Chinese language and the development tendency of related modernization more clearly.

\section{OVERVIEW OF CHINESE GRAMMATICAL FEATURES}

Chinese language has gradually become one of the farreaching languages with its long history and rich connotations. Grammar is an essential part in the study of Chinese. At the same time, to make the Chinese grammatical features clear benefits the teaching as well as daily studying and communication. Therefore, it is necessary to fully understand and master the features of Chinese grammar. In the research of
Chinese grammatical features, different researchers had different views and ideas on it and thereby generated different research results. The author summarizes the Chinese grammatical features as follows.

\section{A. Multi-functionalization of parts of speech in Chinese}

Different from the Indo-European, there is not a simple corresponding relation between the part of speech and the syntactic constituent in Chinese, which can be interpreted as that there is no corresponding relationship between verbs and the predicate, between nouns and the subject or object, between adjectives and the attributive, and so on. The relationship between the part of speech and syntax in Chinese is obviously more complicated than that of Indo-European. Specifically, the subject and object in Chinese can not only be associated with nouns and verbs, but can also be associated with adjectives and adverbs. This means that in Chinese, verbs or adjectives can serve as the subject and object while their morphological shape is as the same as the predicate, But in English, German and other languages, a verb or an adjective must experience the word formation according to special morphology or syntax before it serves as the subject or object. For example, only a verb has been transformed into a noun-form (namely a gerund) can it appears at the position of the subject or object in an English sentence [2].

In the Indo-European, adjectives are often used to modify nouns, and the position of the noun is relatively fixed and cannot directly be used as the attributive. But there is no similar hard-and-fast rule requirement in Chinese. In Chinese, as long as the literal meaning of a noun does not conflict with each other, the noun can act as the attributive while not does affecting the role play of the original subject and object. The weak connection between the part of speech and syntax in Chinese determines the loosening and freedom of Chinese language system to a great extent, which makes the study of grammar more convenient and relaxed. The feature of Chinese of this kind is also one of the prominent attributes of the SinoTibetan.

\section{B. Emphasis on the application and presentation of context}

Context is of great significance to the use of Chinese. In practical applications, the topic is generally put at the beginning of the sentence in Chinese. The specific thing is usually regarded as the main topic in the specific application of Chinese and more likely to be placed at the position of the subject in a sentence, so that the receiver of the language can 
better understand the language information. At the same time, Chinese also shows the combination of long sentences and short sentences in the process of application. But the sentences of this type in Chinese are different from those in English, such as some short sentences like "Who is Xiaowang?" and "I am Xiaowang." There are also short sentences in English and they have the same effect in information delivery. But, in the composition of actual syntax in Chinese, the same expression or part of speech can play the role of different components in different sentence patterns. This is the prominent feature of Chinese language caused by the emphasis on context. In order to express the grasp of the characteristics of language application in a specific context, Chinese is usually inflexible in form and characterized by better use. At the same time, the prosody is also highlighted in the application of Chinese and the use of syntactic structure can be well controlled by rhythm.

\section{Analytical characteristics and lack of obvious part of speech transition}

In practical application, Chinese is an obvious analytical language system and lacks morphological changes in the standard sense, which can be seen from the lack of abundant and figurative part of speech change marks. The reflection of tense is also not obvious in Chinese. Unlike English, Chinese does not explicitly emphasize the specific syntax and words of a tense, and thus it shows the loosening of structure and lack of symbols marking tense and voice [3]. At the same time, Chinese grammar shows consistency in the construction and expression of compound words. The Chinese has relatively uniform and regular structure, and also shows a better semantic and environmental fusion in the actual use process.

\section{THE MODERNIZATION OF CHINESE LINGUISTICS}

Chinese linguistics is a subject devoted to the study and analysis of Chinese, and the main contents of this discipline include the lexical, syntactic, semantic and contextual features of Chinese as well as differences with other languages. To some extent, it introduces the historical evolution and cultural background of Chinese, highlighting the important role of Chinese as the traditional language of the Chinese nation and the prominent role of communication culture.

The process of modernization of Chinese linguistics is inevitably accompanied by collisions and exchanges with foreign cultures and corresponding languages. This is also a problem needing to be investigated in the modernization process of Chinese linguistics. With the increasingly important role and status of China in the process of international communication, to improve the comprehensive development level and language development environment of Chinese linguistics is of great significance to the development of Chinese linguistics. Therefore, the modernization of Chinese linguistics is a comprehensive study based on the application and characteristics of Chinese language, the comprehensive analysis of the world cultural pattern and the situation and development trend and other factors.

\section{A. Development of Chinese linguistics}

1) The combination of Chinese and foreign research methods becomes the mainstream

It is known to all that, in the early years, the study of Chinese language mainly focused on language theory but weakened the study of language application. Through continuous exploration in related fields and the research strength improvement of language talents, the Chinese language study in our country has now turned to the applied research which specifically manifests as the translation between English and Chinese, the translation between Chinese and Japanese, the translation between Chinese and Russian and other forms. Actively translating foreign language products (such as movies, TV shows and comics) into China not only quickly enhances the level of cultural exchanges between China and foreign countries, but also enhances the comprehensive strength of linguistics in China. This perfects the language application and enhances theoretical innovation while promoting the language research, improving the research strength in reality. At the same time, in order to improve the development of Chinese linguistics, the state has established relevant undergraduate programs and encourage researchers to actively explore the fields related to the features as well as application and development of the Chinese language, which well interprets the differences and relationship between Chinese culture and foreign culture, opening a way of innovation for the internationalization of Chinese [4].

\section{2) Lack of fixed research mode}

Chinese research involves a wide range, covering almost all aspects of social life. Therefore, it shows a strong tendency to pertinence research in the actual research. For example, in the research of pragmatics, there are various studies of euphemism, business terms and other aspects based on different service environments. As a result of the different specific content involved, the actual research of Chinese language shows a casual status quo. Chinese has been used in China for a long time, so that most Chinese people think they understand Chinese very well and thereby ignore the practical value of Chinese linguistics as a subject. As a matter of fact, Chinese linguistics, as a comprehensive subject of Chinese studies, largely serves Chinese language application and provides a good scientific platform for the development of Chinese. Therefore, to lay stress on the learning of Chinese language is to attach importance to the future development of Chinese. This has not been paid much attention to in social consciousness and research fields today, and therefore the research model is random and the results are not systematic and useful [5].

Although the process of comprehensive study of Chinese linguistics is not very optimistic, the existing research progress has laid the corresponding foundation for future related research, which provides overarching guidance for the modernization of Chinese in the research on characteristics and application of Chinese linguistics. 


\section{B. Measures to promote the modernization of Chinese linguistics}

Based on the above study and analysis of the characteristics of Chinese grammar and the development of Chinese language modernization, it is easy to found that the present Chinese linguistics is still at the stage of basic descriptive analysis. This is a method applied language analysis, which can improve the understanding and practical skills of beginners of Chinese language. However, the modernization of Chinese linguistics needs a huge comprehensive system, which not only covers the study of all aspects of Chinese language and summary analysis, but also includes the unique interpretation of Chinese language in the light of its development. This can also be counted as a measure and a strategy to promote the development of Chinese linguistics.

\section{1) Paying more attention to the ultimate goal of cultural} communication

In recent years, the study of Chinese linguistics gets an overall rise, and the academic circles are basically carrying on the corresponding theoretical research on the existing basic types of language and cultural heritage. For example, in 1980s, attention began to be paid to the study of the dialects in northwestern Fujian, Northern Shanxi and other places. Ye Mingsheng made a protective countermeasure research on dialects and he held that attention should be given to dialects, as branches of the Chinese; he also believed that its significant role in national culture should be an important part of the comprehensive development of modern Chinese system. The strong regional features and customs in styles of Chinese language are the comprehensive expressions of regional culture and customs.

Seen from the present status of foreign language research, the role of language as a bridge of cultural communication is becoming more and more obvious with the deepening of international communication. An good atmosphere for the learning and study of Chinese contributes to the further development of Chinese, so as to cope with impacts of the foreign language and culture on Chinese traditional culture and ultimately achieve friendly exchanges and mutual promotion between Chinese and foreign cultures. Therefore, more attention should be given to the basic purpose of cultural communication in the future research of Chinese linguistics. In the study, the language application should be placed in the first place to complete the internationalized application of Chinese under the guidance of the existing cultural theories, translation linguistics and other theories. Taking the popular Lecture of Chinese Culture and the Confucius Institute as the instance, they spread the Chinese culture and bring the language charm of Chinese around the world. At the same time, they also introduce languages with more foreign attributes into China's academic circles for study and analysis, achieving a good blend of Chinese and foreign languages and cultures and also promoting the deep development of language research and learning. It is foreseeable that, in the process of cultural exchange between China and foreign countries, the role of language as a pioneer will receive great attention in the future. In order to better use Chinese as a tool for international communication and achieve the modernization of Chinese, it is necessary to conduct corresponding research and analysis premised on specific objectives, ultimately aiming to strive to make targeted developmental planning after understanding allround problems (such as the grammar environment, cultural atmosphere and application rules of Chinese language) to achieve the modernization.

2) Laying stress on the important role of school in language research

The school is an important comprehensive learning site for language studies. School education has irreplaceable function for scholars and experts engaged in language studies, so it is necessary to link up with the organization or education in the real Chinese research activities. To this end, colleges and universities need to actively carry out education and training activities, including playing videos with teaching and guiding significance, imparting knowledge of related books and organizing targeted interpretations presented by scholars with rich experience in foreign language teaching and research, and then to realize the norms of language research. In addition, colleges and universities should often communicate with language scholars to understand the needs of language research for a period of time, so as to constantly innovate the content and way of research, rather than just being limited to traditional theoretical research.

At the same time, the school should strengthen the actual investment in Chinese teaching -- including investment in both teaching resources and scientific research resources -- to deepen its important role in the dissemination of Chinese language, enhance students' understanding of the modernization of Chinese linguistics, promote their thinking on the development of Chinese Linguistics in a actual atmosphere of Chinese language and culture research, deepen their understanding of the Chinese language and culture as well as corresponding grammatical features and study the comprehensive application and system, ultimately promoting the construction of modernization of Chinese language.

\section{CONCLUSION}

To sum up, as a bridge and link between Chinese and foreign cultures, the research of foreign languages must be completed based on the actual needs. However, the actual efficiency of foreign language research in China under the traditional concept is not good due to the heterogeneous operating environment and complicated semantics of foreign languages. In the future, the research and application of foreign languages will be related to the whole language research environment and the actual exchange of Chinese and foreign cultures. Therefore, it is necessary to innovate research methods, pay attention to the practical purpose of research and improve the concrete methods of research. Only in this way can the research work of foreign languages be promoted.

\section{REFERENCES}

[1] Su Zhanghai. A New Achievement of Chinese Linguistics from the Perspective of Contrastive Study -- Essentials in SBU Theory [J]. Hebei Normal University of Science \& Technology (SOCIAL SCIENCE), 2016,15 (01): 88-98. 
[2] Xu Jie. Few Affixes but Many Clitics -- a Re-summary of Chinese Grammatical Features [J]. Journal of Huazhong Normal University (HUMANITIES AND SOCIAL SCIENCES), 2012,51 (02): 113-118.

[3] He Yingying. Discussion on the Characteristics of Chinese Grammar and the Modernization of Chinese Linguistics [J]. Intelligence, 2014, (06): 254.
[4] Xin Xueying. On the Diachronic development of verb reduplication and Its Grammatical Features in Modern Chinese [D]. Shaanxi Normal University, 2016.

[5] Ma Xiaoxiao. Characteristic Analysis of the Israel Country Specific Chinese Textbook Modern Chinese Grammar [D]. Beijing Foreign Studies University, 2015. 